\title{
STATUS OF ADOLESCENTS WITH SYMPTOMS OF IRON DEFICIENCY IN UKRAINE
}

D0I: 10.36740/WLek202003127

\author{
Larysa F. Matiukha, luliia S. Perig, Oleksandr Ye. Kononov \\ DEPARTMENT OF FAMILY MEDICINE AND OUTPATIENT CARE, SHUPIK NATIONAL MEDICAL ACADEMY OF POSTGRADUATE EDUCATION, KYIV, UKRAINE
}

\begin{abstract}
The aim: The purpose of this research was to study the reasons adolescents are underdiagnosed in iron deficiency in Ukraine.

Materials and methods: We provided a data analysis of medical records on the reasons and frequency of requests for medical assistance among teenage children, including the age group 10-19 years. The group of exclusion criteria included patients with traumatic brain injury, burns, eye diseases, congenital abnormalities of development, childhood infirmities, persons who had contact with radiation, oncological patients, patients who had surgery within the last 3 months. Moreover, the researched cases were provided by private medical institutions as we needed to compare the laboratory tests, which are not available in governmental primary care institutions. In this research were used such key methods as basic data analysis, correlation-analysis and summarizing of gathered results.

Results: The number of referrals dominated initially to ENT (36.8\%), family physicians (25.6\%) and pediatricians (23.3\%), with a nearly equal distribution of patients by gender. From all the cases there were 162 cases where clinical laboratory blood test was performed (56,8\% male, $43,2 \%$ female). The distribution among specialties of doctors, who prescribed a blood test was: $64,2 \%$ family doctors, $9,3 \%$ ENT, $20,4 \%$ pediatricians, $6,1 \%$ - other specialists. Serum ferritin was discovered in 22 cases. They were found in $19,1 \%$ and there was no correlation between inflammation changes and number of erythrocytes, hemoglobin and qualitative signs of red blood cells.

Conclusions: The analysis of medical records revealed the expediency of the syndromic distribution of causes of treatment, as well as the identification of risk groups and early diagnosis of pathologies among adolescent children in the practice of a family doctor. A fifth part of adolescent patients appointments was made up of healthy individuals, of whom (69.5\%) belonged to the age group of 10-13 years. Inflammatory changes in the blood are not related to the amount of hemoglobin, erythrocytes and erythrocyte characteristics. Hemoglobin, hematocrit and erythrocyte counts are not informative in the diagnosis of iron deficiency.
\end{abstract}

KEY WORDS: iron deficiency, hemoglobin, hematocrit, adolescent.

Wiad Lek. 2020;73(3):546-550

\section{INTRODUCTION}

Family doctor (FD) faces a wide variety of pathologies in his everyday practice. Nowadays, in the realities of health care reform, primary care plays the leading and essential role in guiding patients to early diagnosting, treating, preventing diseases. Almost $80 \%$ of patients start and end their pre-hospital treatment not being carried to the secondary medical care. According to this fact, it is important to know risk groups of population to each abnormal condition of health. WHO provided a report "Health for the world's adolescents" fully addressing that question across the broad range of health needs of people ages $10-19$ years and found them as a core area for health sector action. [1-3] National Center of Statictic in Ukraine shows, that in 2019 year there are nearly 13 million in rural areas and 29 million in cities of population, where part of people aged 10-19 years amounts 750 thousand in rural areas and 1,5 million in cities. For last 10 years morbidity in Ukraine remains nearly at the same levels. Adolescents usually get medical care in pediatricians or family doctors and the volume of diagnostical tools and services can differ depending from territory of living, region, whether it is governmental or private clinic, and for sure varies depending on knowledge and skills of doctor. Iron deficiency and anemia is one of the most common public health difficulties in the world and in Ukraine as well. [1-4] ID is associated with intencive cognitive function, impaired physical work capacity, physiological changes in hormones and poor pregnancy outcomes. The onset of menstruation in girls, rapid growth with phycological conditions that occur during adolescence, and the consumption of predominantly plant-based diets with low bioavailable iron, as well as product which tend to decrease iron consumption, all contributes to the depletion of iron stores that substantially increases the risk of teenage population to ID. What is more, the compliance to oral iron supplementation keeps quite low posissions because of side-effects of medicines, therefore it is important to diagnose ID as early as possible. $[3,5]$

\section{THE AIM}

The purpose of this research was to study the reasons adolescents are underdiagnosed in iron deficiency in Ukraine.

\section{MATERIALS AND METHODS}

We provided a data analysis of medical records on the reasons and frequency of requests for medical assistance 


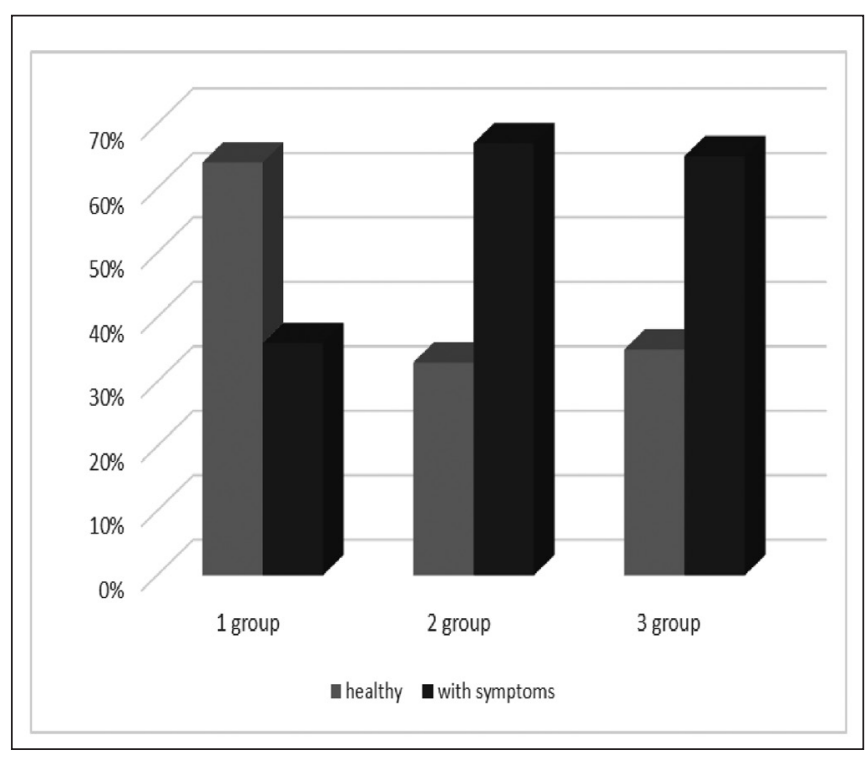

Fig. 1. Distribution among generally healthy teenagers and with deseases.

among teenage children, including the age group 10-19 years in Kyiv during 2018. The group of exclusion criteria included patients with traumatic brain injury, burns, eye diseases, congenital abnormalities of development, child- hood infirmities, persons who had contact with radiation, oncological patients, patients who had surgery within the last 3 months. Moreover, the researched cases were provided by private medical institutions as we needed to compare the laboratory tests, which are not available in governmental primary care institutions.

In this research were used such key methods as basic data analysis, correlation-analysis and summarizing of gathered results.

The work was performed in accordance with the principles of the Helsinki Declaration of the World Medical Association "Ethical principles of medical research with the participation of a person as a research object" and approved by the Bioethics Commission of Shupik National Medical Academy of Postgraduate Education.

\section{RESULTS}

According to the World Health Organization (2007) anemia in adolesents is defined as hemoglobin level $<12.0$ $\mathrm{g} / \mathrm{dl}$. in female after 11 y.o and $<13.0 \mathrm{~g} / \mathrm{dl}$ in men in the age-group after 15 y.o. Serum ferritin level $<15 \mu \mathrm{g} / \mathrm{L}$ and soluble transferrin receptor (sTfR) level $<5.0 \mathrm{mg} / \mathrm{L}$ were considered as iron deficiency status.[6-7]

There were 725 cases of the primary application to the doctor for medical aid 390 (53.8\%) female and 335 (46.2\%)

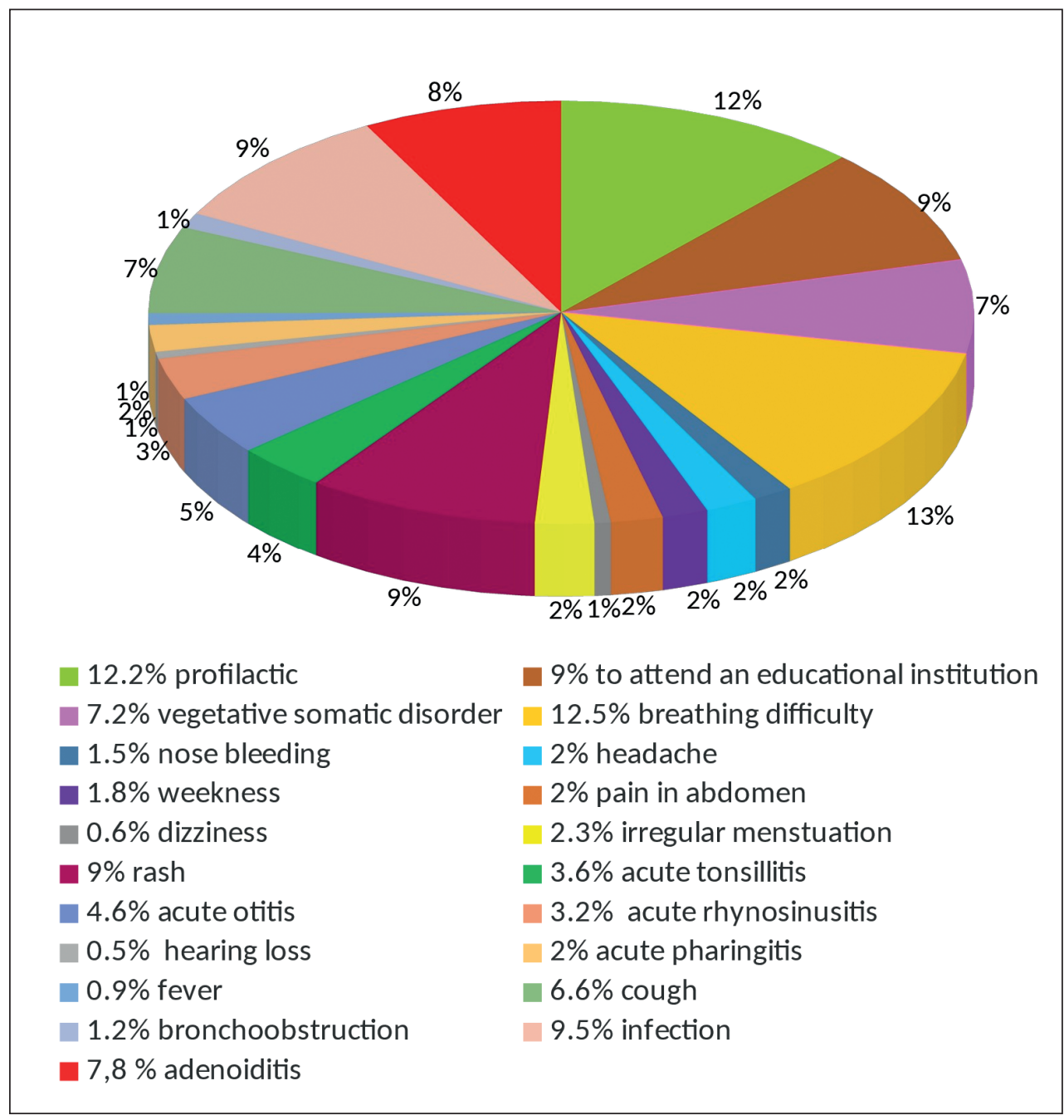

Fig. 2. Causes for appointment to doctor in patients aged 10-19 y.0. 


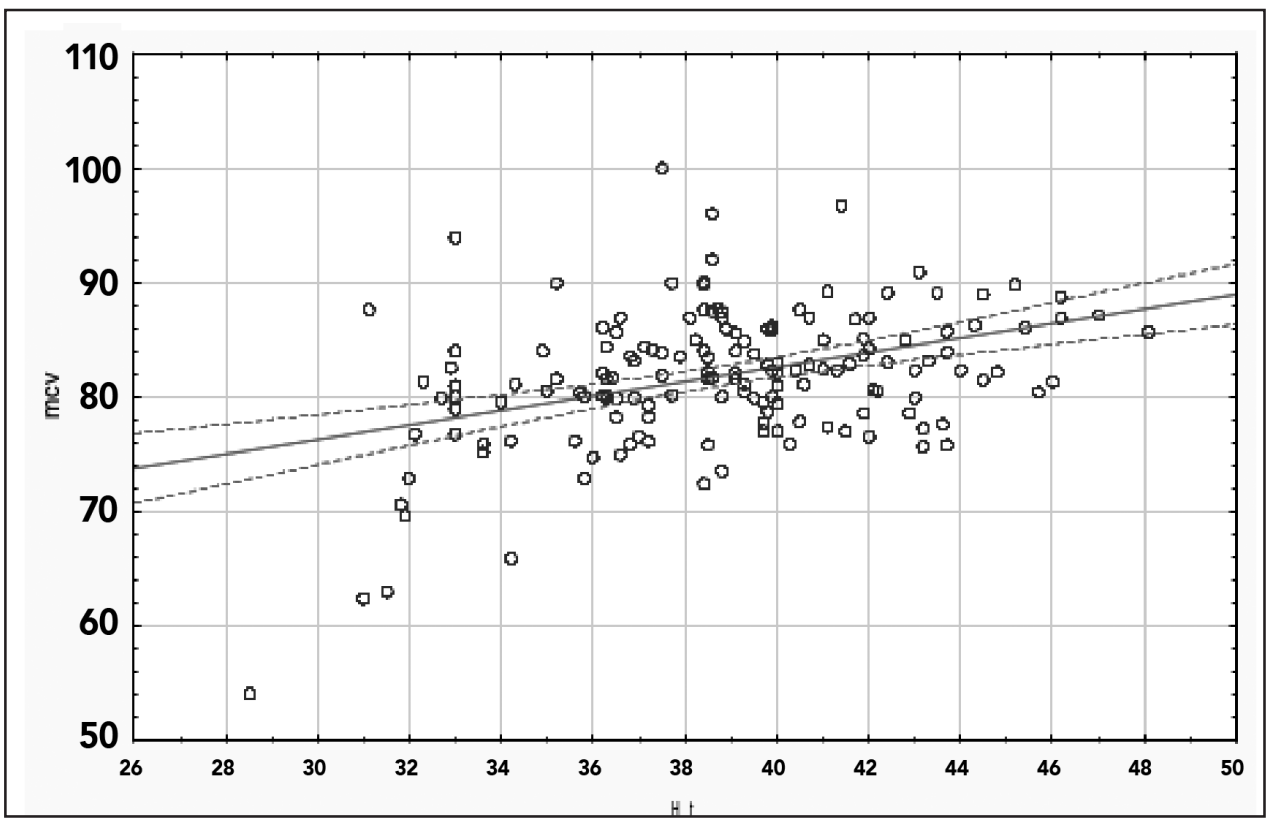

Fig. 3. Correlation between Ht and MCV

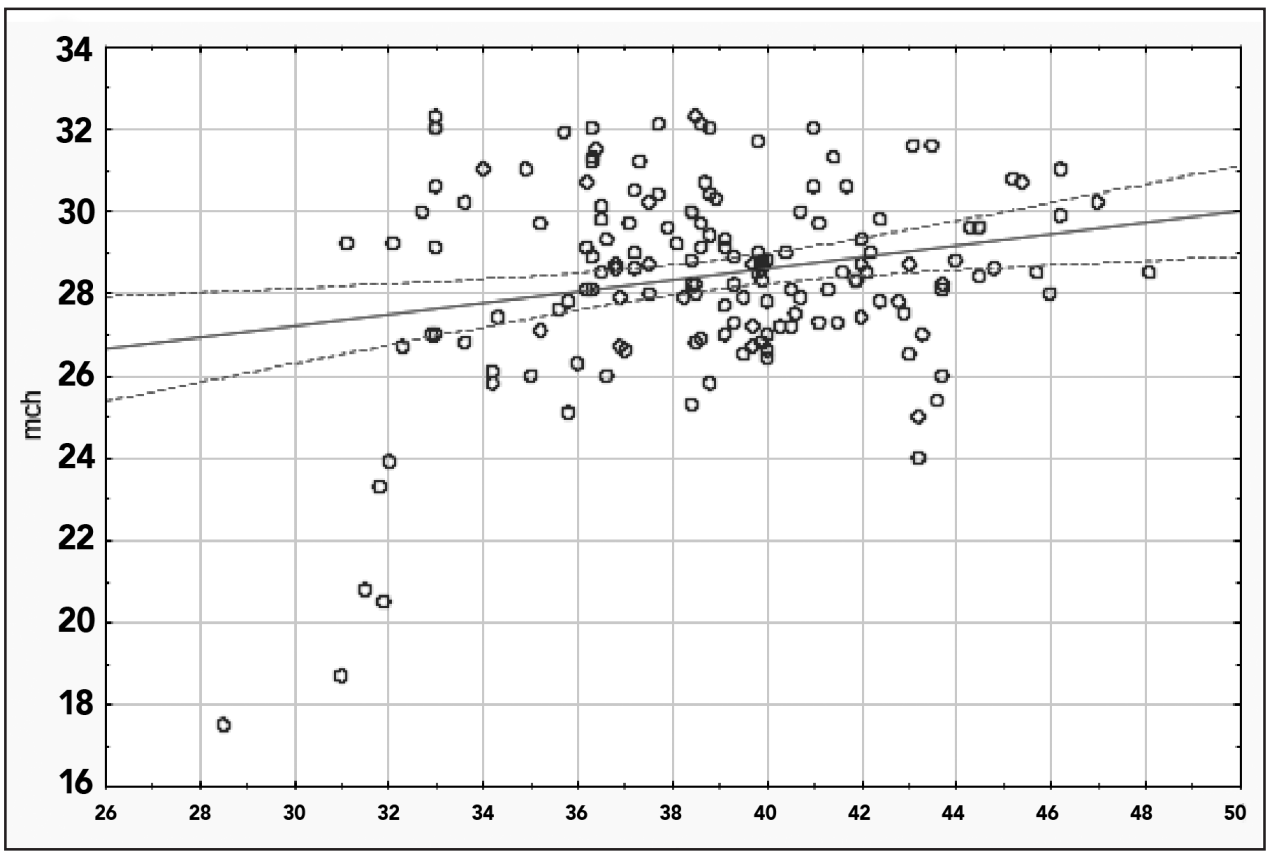

Fig. 4. Correlation between $\mathrm{Ht}$ and $\mathrm{MCH}$

male 2018. The distribution according to the age was: early adolescent age (10-13 y.o.) group 1 - 338 cases (185 female, 153 male), middle adolescent age (14-17 y.o.) group $2-365$ cases (189 female, 176 male) and late adolescent age (18-19y.o.) group $3-22$ cases ( 16 female, 6 male). The comparison above shows approximately equal percentages of male and female adolescents in groups 1 and 2. More than fifteen times less than among adolescents of late age. All the reasons of primary appointment to doctors were divided into two categories: mostly healthy individuals who need a medical conclusion for some activities or attending educational institutions or patients who needed a compulsory annual medical examinations because of complaints. We found that the quantity of in generall healthy teenagers was among all $21.2 \%$, who mostly were people from group 1 (early adolescent age). The primary morbidity and acute pathology were higher in the $2^{\text {nd }}$ group, but in general in numbered $57,6 \%$. It is notable, that morbidity in the $3^{\text {rd }}$ group was nearly the same as in $2^{\text {nd }}$ one. (Figure 1)

The number of referrals dominated initially to ENT (36.8\%), family physicians $(25.6 \%)$ and pediatricians $(23.3 \%)$, with a nearly equal distribution of patients by gender. There was a difference of $29.4 \%$ with a predominance of male among the consultations with a neurologist, $9 \%$ with a predominance of a female with a referral to a gastroenterologist and similarly with $12.4 \%$ with a referral to a dermatologist.

From all the cases there were 162 cases $(22,3 \%)$ where clinical laboratory blood test was performed (56,8\% male, 
$43,2 \%$ female). The reason why they've got referrals were 78 patients (48\%) - routine yearly examination, 11 patients $(6,79 \%)$ - clinical iron deficiency symptoms, 73 (45,21\%) on other reasons or suspicion to inflammation. (Figure 2)

The distribution among specialties of doctors, who prescribed a blood test was: $104(64,2 \%)$ family doctors, 15 (9,3\%) ENT, $33(20,4 \%)$ pediatricians, $10(6,1 \%)$ - other specialists. Serum ferritin was discovered in 22 cases $(13,5 \%)$. Inflammatory changes were considered if the number of leukocytes was high and erythrocyte sedimentation rate higher than $15 \mathrm{mmol} / \mathrm{l}$. They were found in 31 cases $(19,1 \%)$ and there was no correlation between inflammation changes and number of erythrocytes (Er), hemoglobin $(\mathrm{Hb})$ and qualitative signs of red blood cells. $\mathrm{MCV}<80$ was found in 45 cases, of these, the hemoglobin was lower then $120 \mathrm{~g} / \mathrm{l}$ in 7 cases, Er under normal age point - in 2 cases. $\mathrm{MCH}<27$ was found in 35 cases, of these, the hemoglobin was lower then $120 \mathrm{~g} / \mathrm{l}$ in 11 people, Er under normal age point - in 1 case. Analyzes containing hematocrit and color index were obtained in 79 cases ( $48.76 \%$ of all analyzes), in other cases there was no color index and hematocrit. The result distribution: : hemoglobin $135 \mathrm{~g} / 1$ ( \pm 15.5), erythrocyte count $4.9( \pm 0.67)$, hematocrit $39.57( \pm 4)$, platelet count 264 ( \pm 68.5$)$, leukocyte count 7.39 ( \pm 2.79$)$, MCV $81.6( \pm 6.59), \mathrm{MCH} 27.95$ ( \pm 2.47$)$, color index (CP) 0.84 $( \pm 0.07)$. (Figure 3, figure 4)

According to the correlation analysis of indicators of clinical blood analysis revealed that there is a direct strong correlation between hemoglobin level and hematocrit $(\mathrm{r}=$ $0,94)$, a direct significant correlation between hemoglobin level and $\mathrm{MCH}(\mathrm{r}=0,63)$ and color index $(\mathrm{r}=0.63)$, a direct moderate relationship between hemoglobin level and erythrocyte count $(r=0.45)$ and MCV $(r=0.49)$. There is a direct very strong correlation between color index and $\mathrm{MCH}(\mathrm{r}=0.93)$ and $\mathrm{MCV}(\mathrm{r}=0.9)$, a direct strong relationship between $\mathrm{MCH}$ and $\mathrm{MCV}(\mathrm{r}=0.88)$, a direct significant relationship relationship between hematocrit level and MCH $(\mathrm{r}=0.58), \mathrm{MCV}(\mathrm{r}=0.56)$ and color index $(\mathrm{r}=0.57)$.

\section{DISCUSSION}

This research was conducted as a part of $\mathrm{PhD}$ research in social medicine according to the topic of iron deficiency screening. Sample size was considered as significant if there were more then 400 cases discovered. The exclusion criteria patients with traumatic brain injury, burns, eye diseases, congenital abnormalities of development, childhood infirmities, persons who had contact with radiation, oncological patients, patients who had surgery within the last 3 months. Randomization was generated hence we provided our analysis in different districts, didn't deal with patients, we only worked with their former conclusions, during 2018.

The results of correlation analysis should be proved in a bigger sample size and to compare the results with ferritin level, as well as it's essential to prove the results in different regions of Ukraine.
The analysis of medical records revealed the expediency of the syndromic distribution of causes of treatment, as well as the identification of risk groups and early diagnosis of pathologies among adolescent children in the practice of a family doctor. There is a tendency for the highest number of complaints about diseases of the upper respiratory tract (46.4\%), skin and subcutaneous tissue (9\%), vegetative somatic disorders $(7.2 \%)$. A fifth part of adolescent patients appointments was made up of healthy individuals, of whom (69.5\%) belonged to the age group of 10-13 years. In the age group of $14-17$ years the figure was (28.7\%). The majority of appeals among children aged 10-19 were appeals to the ENT doctor (36.8\%), family doctor (25.9\%), pediatrician $(23.3 \%)$. The distribution among specialties of doctors, who prescribed a blood test was: $104(64,2 \%)$ family doctors, 15 (9,3\%) ENT, 33 (20,4\%) pediatricians, $10(6,1 \%)$ - other specialists. Serum ferritin was discovered in 22 cases $(13,5 \%)$.

\section{CONCLUSION}

Inflammatory changes in the blood are not related to the amount of hemoglobin, erythrocytes and erythrocyte characteristics. Hemoglobin, hematocrit and erythrocyte counts are not informative in the diagnosis of iron deficiency. Consequently, patients of the adolescent age need to be yearly referred to the clinical blood test with checking ferritin if there are any risks of iron deficiency. In the case, when there are no MCH and MCV mentioned, it is possible to consider color index as marker of possible iron insufficiency.

\section{REFERENCES}

1. Adolescenthealth:its promise and potential (PartnerSymposium), Berlin, World Health Summit, 2011 URL: https://www.worldhealthsummit. org/fileadmin/user_upload/downloads/WHS_2011_Program_-_ Screen_Version.pdf

2. Sixty-fourth World Health Assembly. Resolution WHA 64.28: Youth and health risks. Geneva, World Health Organization, 2011 URL: http://apps. who.int/gb/ebwha/pdf_files/WHA64/A64_R28-en.pdf

3. Patton GC et al. Next steps for adolescent health: a Lancet Commission, The Lancet. 2014; 383:385-6

4. Patton $G$ et al. Health of the world's adolescents: a synthesis of internationally comparable data. The Lancet. 2012;379(9826):1665-75.

5. Deogan C, Ferguson J, Stenberg K. Resource needs for adolescent friendly health services: estimates for 74 low- and middle-income countries. PLOS ONE. 2012; 7(12):514-520.

6. World Health Organization. Serum Ferritin Concentrations for the Assessment of Iron Status and Iron Deficiency in Populations. Vitamin and Mineral Information System. Geneva, Switzerland: World Health Organization, 2018 URL: http://www.who.int/vmnis/indicators/ serum_ferritin.pdf.

7. World Health Organization. Serum Transferrin Receptor Levels for the Assessment of Iron Status and Iron Deficiency in Populations. Vitamin and Mineral Information System. Geneva, Switzerland: World Health Organization, 2018 URL: http://www.apps.who.int/ iris/bitstream/10665/133707/1/WH0_NMH_NHD_EPG_14.6_ eng. pdf. 
The article is a fragment of the scientific research work "Scientific substantiation of modern approaches to optimization of preventive directions at the primary level of providing medical care" (deadline - 2018-2022, state registration number 0113U002455).

\section{ORCID and contribuntionship:}

Larysa F. Matiukha - 0000-0001-8249-8583 ${ }^{A, F}$

Iuliia S. Perig - 0000-0002-2821-2078 ${ }^{B, C, D}$

Oleksandr Ye. Kononov - 0000-0003-0505-0296 A, E

\section{Conflict of interests:}

The Authors declare no conflict of interest.

\section{CORRESPONDING AUTHOR}

\section{luliia S. Perig}

Zh. Zhabaeva 7d-69, 04112, Kyiv, Ukraine

tel: +380980538584 ,

e-mail: iuliia.perig2506@gmail.com

Received: 17.01 .2020

Accepted: 05.03.2020

A - Work concept and design, B - Data collection and analysis, C - Responsibility for statistical analysis,

D-Writing the article, $\mathbf{E}$-Critical review, $\mathbf{F}$ - Final approval of the article 